\title{
Bayesian correlation between temperature and blossom onset data
}

\author{
Volker Dose* \\ Centre for Interdisciplinary Plasma Science, \\ Max-Planck-Institut für Plasmaphysik, \\ EURATOM Association, Boltzmannstrasse 2, \\ D-85748 Garching bei München, Germany \\ Annette Menzel \\ Department of Ecology, Center of Life Science, TU Munich, \\ Am Hochanger 13, D-85354 Freising, Germany
}

(Dated: February 27, 2006)

Keywords: Bayesian theory, phenology, temperature, climate change

Running title: climate change relevance to phenology

\footnotetext{
* Corresponding author: Prof. Dr. Volker Dose, Centre for Interdisciplinary Plasma Science, Max-PlanckInstitut für Plasmaphysik, Boltzmannstrasse 2, D-85748 Garching bei München, Germany, Tel: +49-893299-1265, Fax: +49-89-3299-2591, Email: volker.dose@ipp.mpg.de
} 


\section{Abstract}

The recent quantification of changes in time series of phenology data with Bayesian methods has provided compelling evidence for changes during the last twenty years. In this paper we correlate the phenological observations with spring temperature time series. We provide quantitative answers to the question whether changes in temperature and phenological time series should be regarded as coherent or independent. For the three considered species snowdrops, cherry and lime tree we find factors of 1.05, 2.19 and 3.26 respectively in favor of coherence. The functional behaviour and the trend in the temperature time series are presented. They amount to $0.15^{\circ} \mathrm{C} /$ year for the January - March average, $0.09^{\circ} \mathrm{C} /$ year for February - April and $0.1^{\circ} \mathrm{C} /$ year for March - May in 2002. In addition, we compare blossom trends for the coherent and independent hypotheses and find that the transition from trend values slightly positive before 1970 to strongly negative at present becomes sharper as the temperature data is included in the analysis. 


\section{INTRODUCTION}

Observations of phenological phases is probably the simplest way to track changes in the ecology of species in response to climate change. The use of phenological data as bio-indicator for climate variations and global change is based on the well known relationship between climate parameters and the onset of phenological phases. It was Reaumur, who suggested in 1735 that differences between years and locations in the date of phenological events could be explained by differences in temperatures from an arbitrary date to the date of the phenological event considered. This is probably the first mentioning of a relationship between phenological spring phases, such as leaf unfolding and flowering, and spring temperatures. However, phenophases are regarded as an integrating climatological measurement (Schnelle 1955, Menzel 2002) responding to many meteorological and environmental factors such as light, photoperiod, temperature, precipitation, humidity, wind, and others - though their particular influence differs: The timing of leaf unfolding of trees is mainly regulated by temperature, specifically chilling temperatures break winter dormancy and subsequent warm temperatures induce budburst. Photoperiod may play a major role for flowering, however, without interaction with temperature it cannot explain the annual variability at a given location (Chuine et al. 2003). The phenological onset of spring and summer (leaf unfolding, flowering, fruit maturation) seems to correlate with the temperature of the preceding months. By contrast, various environmental factors are supposed to drive autumn phenology but none of the existing models can predict leaf colouring up to now.

Many publications of recent years have pointed to coherence of phenological spring phases and temperature either by classical statistical methods (correlation analysis, linear and multiple regression) (e.g. Boyer 1973, Sparks \& Carey 1995, Emberlin et al. 1997, Sparks et al. 2000, Fitter \& Fitter 2002, Menzel 2003) or fitting and testing statistical models mainly based on temperature (e.g. Schwartz 1997). The complexity increases in more mechanistically formulated models incorporating chilling and forcing, both based on temperature (e.g. Landsberg 1974, Cannell \& Smith 1983, Hänninen 1987, Murray et al. 1989, Kramer 1994, Chuine 2000, Linkosalo et al. 2000, see reviews by Hänninen 1990 and Chuine et al. 2003). Most recently, new approaches on a physiological basis use promoting and inhibiting variables which are related to temperature (Schaber \& Badeck 2003). Experiments are another possibility to demonstrate that phenological spring phases are mainly triggered by 
temperature (and photoperiod).

Tests of phenological models by cross validation exhibit fairly good results, tests of their predictive power in carbon enrichment / warming experiments sometimes produce unexpected results (Hänninen et al. 1993).

The relationship between spring phenology and temperature enables the use of phenology as bio-indicator for climate change (e.g. as suggested by the European Environmental Agency, see Menzel 2002). Within the Third Assessment Report of the IPCC (2001) results of publications revealing climate change impacts were included when the study matched 2 of 3 criteria: 1) temperature change over the period, 2) changed impact variable over the time, 3) proved relationship between temperature and variable. This paper focusses on the first and third of these criteria and derives Bayesian measures for the relationship between blossom onset and temperature. Readers who are unfamiliar with Bayesian ideas are referred to the excellent tutorial by Sivia (1996).

Our knowledge that spring phenology is primarily driven by temperature prompts us to attribute observed biological trends to effects of climate variations when we demonstrate a high statistical coherence between temperature and onset of phenological phases, as suggested by many papers. In addition to physiologically based model frame work, experiments, and traditional statistical approaches, the relation between temperature data and blossom onset data can be analyzed employing Bayesian probability theory. This approach allows a rigorous analysis of the causal interdependence of phenology and temperature observations. The results of such an analysis are numerical values of the probabilities for the two alternative hypotheses 1.) the evolution of the temperature and blossom time series is independent and 2.) there is coherence in the evolution of blossom onset data and temperature time series.

\section{MATERIAL}

Three long-term flowering records (1896-2002), observed at the Research Station of the German Weather Service at Geisenheim (49 $\left.59^{\prime} \mathrm{N}, 7^{\circ} 58^{\prime} \mathrm{E}\right)$, have been analysed comprising first flowering of snowdrops (Galanthus nivalis L.), of sweet cherry (Prunus avium L.), and of lime tree (Tilia platyphyllos L.). They were also the basis of the analysis in the preceding paper (Dose \& Menzel 2004). Mean monthly air temperatures were available for the period 
1935-2002 from the adjacent climate station of Geisenheim research station for which no inhomogeneities are known, such as a displacement of the climate station or varying urban heat island effects. The two sets of data stem from the same place which justifies their use in the analysis described in this paper. In order to avoid later ambiguities: the blossom time series consists of the day of blossom onset in a given year. The temperature time series consists also of one entry per year which is obtained from monthly averages characteristic for each of the three species which will be considered in this paper.

\section{TEMPERATURE TIME SERIES}

In analogy to the procedure adopted to describe blossom time series (Dose \& Menzel 2004) we shall employ a two section polygonial to model temperature time series. The rational for this choice is that such a triangular function is the simplest assumption beyond a constant or a linear time dependence of the assumed temperature evolution. Moreover, it was shown previously (Dose \& Menzel 2004) for blossom onset time series that this model is by far more probable than the simpler linear or constant models. The model function is supported at the beginning of the time series in year $x_{1}$ and assumes there the functional value $f_{1}$, at the endpoint of the time series in year $x_{N}$ and assumes there the functional value $f_{N}$. While the endpoints of the time series remain fixed in the subsequent calculations the intermediate point $x_{E}$ with associated functional value $f_{E}$ can assume all years $x_{2} \leq x_{E} \leq x_{N-1}$. We

shall call the vector $\vec{f}=\left[f_{1}, f_{E}, f_{N}\right]^{T}$ the support functional values. The support functional values, as well as the matching point of the two linear sections making up the triangular function are variables of the calculation. Let $x_{k}$ denote the year of observation, $d_{k}$ the data collected in year $x_{k}$, and $\epsilon_{k}$ the uncertainty associated with data $d_{k}$, then

$$
d_{k}-\epsilon_{k}=\left\{\begin{array}{l}
f_{1} \cdot \frac{x_{E}-x_{k}}{x_{E}-x_{1}}+f_{E} \frac{x_{k}-x_{1}}{x_{E}-x_{1}}, x_{k} \leq x_{E} \\
f_{E} \cdot \frac{x_{N}-x_{k}}{x_{N}-x_{E}}+f_{N} \frac{x_{k}-x_{E}}{x_{N}-x_{E}}, x_{k} \geq x_{E}
\end{array} .\right.
$$

The matching position of the two linear segments is $x_{E}$ and will, henceforth, be called matching point. Index $k$ runs over all $N$ observations. It is convenient to rewrite (1) in matrix notation

$$
\vec{d}-\mathbf{A}(E) \vec{f}=\vec{\epsilon}
$$

Matrix A is a three column matrix with $N$ rows, where $N$ is the number of observations. The rows of $\mathbf{A}$ have as the first element the coefficient of $f_{1}$ in (1) and as the second element 
the coefficient of $f_{E}$ in (1) if $x_{k}<x_{E}$. The third element is zero in this case. Conversely if $x_{k}>x_{E}$ the first element is zero, the second the coefficient of $f_{E}$ in (1) and the third the coefficient of $f_{N}$ in (1). For $x_{k}=x_{E}$ the first and third elements in the row are zero and the second is equal to one.

If the expectation values of the errors $\left\langle\epsilon_{i}>\right.$ in (1) are zero and their variance is assumed to be $\left\langle\epsilon_{i}^{2}\right\rangle=\sigma^{2}$ then by the principle of maximum entropy (Jaynes 2003, 1957; Kapur \& Kesavan 1992) the sampling distribution of the data becomes

$$
p(\vec{d} \mid \vec{x}, \sigma, \vec{f}, E, I)=\left(\frac{1}{\sigma \sqrt{2 \pi}}\right)^{N} \exp \left\{-\frac{1}{2 \sigma^{2}}(\vec{d}-\mathbf{A}(E) \vec{f})^{T}(\vec{d}-\mathbf{A}(E) \vec{f})\right\} .
$$

In terms of the so far unknown conditions $\sigma, \vec{f}, E$ this function is usually called the likelihood of the data. There is also the further condition "I" which enters into all the conditional probabilities densities. It denotes all background information relevant to the respective probabilities which need not be made explicit in the calculations (Sivia 1996, Gregory 2005). It provides the basis for all further inferences employing the product and the marginalization rules and Bayes theorem. Let us first focus on the probability of a matching point position $E$ given the data $\left\{x_{i}, d_{i}\right\}$. From Bayes theorem

$$
p(E \mid \vec{d}, \vec{x}, I)=p(E \mid I) \cdot p(\vec{d} \mid \vec{x}, E, I) / p(\vec{d} \mid \vec{x}, I),
$$

this probability is provided by the prior probability of a matching point position $E, p(E \mid I)$ times the marginal likelihood $p(\vec{d} \mid \vec{x}, E, I)$. The latter is derived from

$$
p(\vec{d} \mid \vec{x}, E, I)=\int d \sigma d \vec{f} p(\vec{d}, \sigma, \vec{f} \mid \vec{x}, E, I) .
$$

Employing the product rule to the integrand this becomes

$$
p(\vec{d} \mid \vec{x}, E, I)=\int d \sigma d \vec{f} p(\sigma \mid I) \cdot p(\vec{f} \mid I) \cdot p(\vec{d} \mid \vec{x}, \sigma, \vec{f}, E, I) .
$$

Evaluation of (6) requires the specification of the prior probabilities $p(\sigma \mid I)$ and $p(\vec{f} \mid I)$. The least informative prior on $\sigma$, which relies only on the transformation invariance requirement of a scale variable is Jeffreys' prior (Jeffreys 1961)

$$
p(\sigma \mid I)=\frac{1}{\sigma}
$$

Our prior knowledge on $\vec{f}$ is also very vague and we express this by assigning a flat prior to $\vec{f}$

$$
p(\vec{f} \mid I)=\left(\frac{1}{\Delta B}\right)^{3}
$$


over some given range $\Delta B . \Delta B$ is of course somewhat smaller than the data range $\Delta B<$ $d_{\max }-d_{\min }$ and sufficiently large that the integration over $\vec{f}$ in $(6)$ can be extended to infinite limits with insignificant effect on the value of the integral. Integrations over $\vec{f}$ and $\sigma$ can then be performed analytically. The probability of a matching point position $E$ follows then from (4) after appropriate specification of the prior probability $p(E \mid I)$. Candidate points for $E$ are all observation times $x_{2} \ldots x_{N-1}$ hence all years of observation excluding the initial and final year. An uninformative choice for the prior probability $p(E \mid I)$ is therefore the constant

$$
p(E \mid I)=\frac{1}{N-2},
$$

where $N$ is the number of years covered by the data. Finally, using

$$
p(\vec{d} \mid \vec{x}, I)=\sum_{E} p(\vec{d}, E \mid \vec{x}, I)=\sum p(E \mid I) \cdot p(\vec{d} \mid \vec{x}, E, I)
$$

we obtain the normalized probability $p(E \mid \vec{d}, \vec{x}, I)$ for a matching point position $E$ as

$$
p(E \mid \vec{d}, \vec{x}, I)=p(E \mid I) p(\vec{d} \mid \vec{x}, E, I) / \sum_{E} p(E \mid I) p(\vec{d} \mid \vec{x}, E, I) .
$$

The top row in Fig. 1 shows this function for temperature averages January - March, February - April and March - May as full squares. Open circles in the second row panels represent the observations $\vec{d}$ from which these probabilities were calculated. The time range covered is from 1935 until 2002. The second row in Fig. 1 shows also our final estimates of the development of the average temperature with time. The uncertainties are also given. The reader might have expected at this point a triangular function. In fact, this would be the maximum likelihood estimate for the problem. The outstanding advantage of the Bayesian approach is that we can get rid of parameters which we need at an intermediate step of the calculation, here, the position of the matching point $x_{E}$, by marginalization. It was shown in detail in (Dose \& Menzel 2004) that the result of this marginalization is the weighted average of all possible $N-2$ triangular functions with weights given by the matching point probabilities shown in the first row of Fig. 1.

The trend shown in the bottom row was obtained by the same procedure with the derivative $d / d x_{i}$ of (1) as the model function. Variances of these estimates are obtained from the posterior estimates of the respective model functions squared.

The results for February - April and March - May are quite similar in shape, note however that the scales for the trend differ by about a factor of 2 . While the current gradient in the 

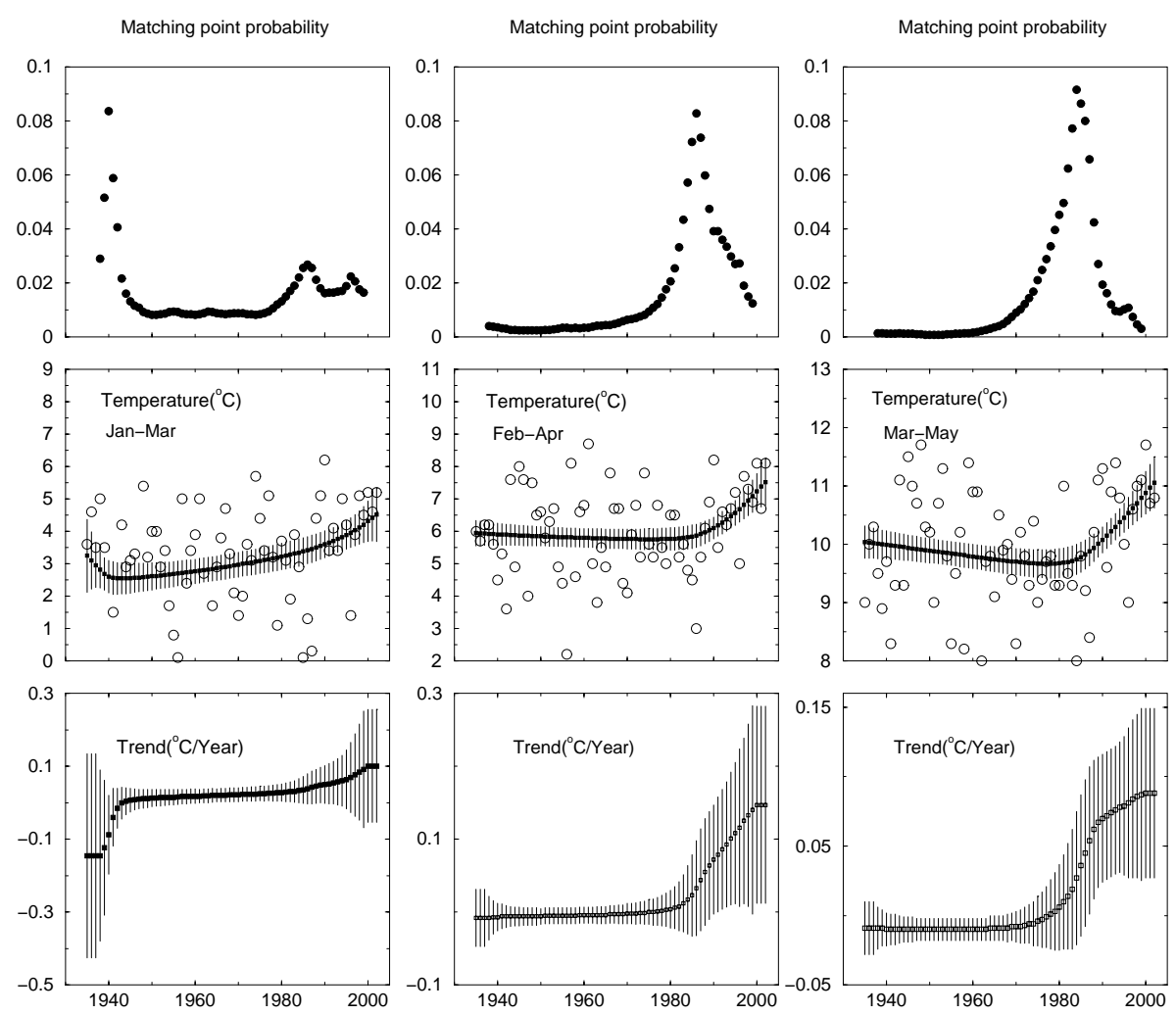

FIG. 1: Open circles in the middle row represent the temperature data. They are three months averages. The full dots in the upper row are the matching point probabilities for a two segment polygonial supported at the ends and at the matching point which we use to model the data. The centre row shows the modeled evolution of the average temperatures with uncertainties based on the data. The lower row exhibits the associated trends. Note that the vertical scales differ between adjacent panels.

February - April trend is about $0.15^{\circ} \mathrm{C} /$ year it is only $0.09{ }^{\circ} \mathrm{C} /$ year for the period March May. The current gradient for January - March is $0.1{ }^{\circ} \mathrm{C} /$ year, the functional behaviour of the temperature differs, however, from the other two cases. While for January - March we 
have a monotonous rise since 1940 with acceleration since 1980 for the other cases we find a weak decrease from 1935 until 1985 with a subsequent strong increase.

\section{RELATION TO BLOSSOM ONSET OBSERVATIONS}

The present identification of matching point probabilities concentrated around the year 1985 bears a strong resemblance to the corresponding matching point probabilities obtained for blossom time series subject to analysis with the same model function (Dose \& Menzel 2004). This raises the question whether we can quantify the hypothesis that the time evolution of the blossom observations can be attributed to the identified temperature changes. This is a simple case of Bayesian model comparison. We compare the two alternative models $M_{a}$ : temperature and blossom onset time series evolved independently and $M_{b}$ : temperature and blossom onset time series exhibit coherence. The results will be probabilities for $M_{a}$ and $M_{b}$ or alternatively the odds ratio of the two probabilities.

Consider first the joint likelihood of temperature data $\vec{d}_{1}$ and blossom data $\vec{d}_{2}$

$$
p\left(\vec{d}_{1}, \vec{d}_{2} \mid \vec{x}_{1}, \vec{x}_{2}, f_{1}, f_{2}, \sigma_{1}, \sigma_{2}, E_{1}, E_{2}, I\right)
$$

It can be decomposed employing the product rule into

$$
\begin{aligned}
& p\left(\vec{d}_{1} \mid \vec{x}_{1}, \vec{x}_{2}, \vec{f}_{1}, \vec{f}_{2}, \sigma_{1}, \sigma_{2}, E_{1}, E_{2}, I\right) \cdot \\
& p\left(\vec{d}_{2} \mid \vec{d}_{1}, \vec{x}_{1}, \vec{x}_{2}, \vec{f}_{1}, \vec{f}_{2}, \vec{\sigma}_{1}, \vec{\sigma}_{2}, E_{1}, E_{2}, I\right) .
\end{aligned}
$$

The messy expression simplifies considerably if we assume logical independence of the data sets $\vec{d}_{1}$ and $\vec{d}_{2}$. This implies in the first place that $p\left(\vec{d}_{2} \mid \vec{d}_{1}, \ldots\right)$ should not be conditional on $\vec{d}_{1}$. In other words, the temperature measurements are not influenced by observations on blossom onset and vice versa. Moreover, the sampling distribution for data $\vec{d}_{i}$ is of course independent of parameters which enter the function modeling $\vec{d}_{k}, \vec{x}_{k}, \vec{f}_{k}, \sigma_{k}, E_{k}$. The joint likelihood for data $\vec{d}_{1}$ and $\vec{d}_{2}$ simplifies therefore to the product

$$
p\left(\vec{d}_{1} \mid \vec{x}_{1}, \vec{f}_{1}, \sigma_{1}, E_{1}, I\right) \cdot p\left(\vec{d}_{2} \mid \vec{x}_{2}, \vec{f}_{2}, \sigma_{2}, E_{2}, I\right)
$$

which provides the basis for the model comparison task. We shall consider two models, $M_{a}, M_{b}$. Let $M_{a}$ assume that the functional behaviour of the blossom time series is not caused by the evolution of temperature. In this case, we have causal independence of the 
two sets of data. This is something entirely different from the above expounded logical independence which allowed to simplify (12) to (14). Neither kind of independence implies the other (Jaynes 2003). In fact, model $M_{b}$ assumes a causal relationin the time evolution of temperature and blossom onset while $M_{a}$ denies it. The key quantity which measures the probability of the respective model is the marginal likelihood

$$
\begin{aligned}
& p\left(\vec{d}_{1}, \vec{d}_{2} \mid \vec{x}_{1}, \vec{x}_{2}, M_{a, b}, I\right)= \\
& \sum_{E_{1}, E_{2}} p\left(E_{1} \mid I\right) \cdot p\left(E_{2} \mid I\right) p\left(\vec{d}_{1} \mid \vec{x}_{1}, E_{1}, I\right) \cdot p\left(\vec{d}_{2} \mid \vec{x}_{2}, E_{2}, I\right) .
\end{aligned}
$$

The explicit form of the factors under the summation sign is given by (6). For model $M_{a}$, which assumes causal independence between the two time series, the marginal likelihood is as given in (15) with independent summation over $E_{1}$ and $E_{2}$. For model $M_{b}$ we obtain a coherent sum over the common matching point position $E$

$$
\begin{aligned}
& p\left(\vec{d}_{1}, \vec{d}_{2} \mid \vec{x}_{1}, \vec{x}_{2}, M_{b}, I\right)= \\
& \sum_{E} p(E \mid I) \cdot p\left(\vec{d}_{1} \mid \vec{x}_{1}, E, I\right) \cdot p\left(\vec{d}_{2} \mid \vec{x}_{2}, E, I\right) .
\end{aligned}
$$

The evaluation of (16) poses a minor problem. Data sets $\vec{d}_{1}$ and $\vec{d}_{2}$ have different lengths. While the blossom data extend over all of the 20th century the temperature data start only in 1935. Two alternatives were considered, truncation of the blossom time series or restriction of the possible matching point positions in $(15,16)$ to the range $1935+1$ until 2002-1. We decided to choose the latter possibility since ignoring precious data seems not appropriate. As a consequence, very tiny differences in the blossom trend shown in the second row of Fig. 2 arise when compared to corresponding graphs of our previous analysis (Dose \& Menzel 2004).

Numerical values of (15) and (16) have been obtained for the January - March temperature/snowdrop blossom, the February - April temperature/cherry blossom, and the March - May temperature/lime tree blossom combinations. Using Bayes' theorem

$$
p\left(M \mid \vec{d}_{1}, \vec{d}_{2}, \vec{x}_{1}, \vec{x}_{2}, I\right) \sim p(M \mid I) \cdot p\left(\vec{d}_{1}, \vec{d}_{2} \mid \vec{x}_{1}, \vec{x}_{2}, M, I\right)
$$

they can be converted to the respective (un-normalized) model probabilities. The posterior odds ratio

$$
\frac{p\left(M_{b} \mid \vec{d}_{1}, \vec{d}_{2}, \vec{x}_{1}, \vec{x}_{2}, I\right)}{p\left(M_{a} \mid \vec{d}_{1}, \vec{d}_{2}, \vec{x}_{1}, \vec{x}_{2}, I\right)}=\frac{p\left(M_{b} \mid I\right)}{p\left(M_{a} \mid I\right)} \cdot \frac{p\left(\vec{d}_{1}, \vec{d}_{2} \mid \vec{x}_{1}, \vec{x}_{2}, M_{b}, I\right)}{p\left(\vec{d}_{1}, \vec{d}_{2} \mid \vec{x}_{1}, \vec{x}_{2}, M_{a}, I\right)}
$$


factors into a prior odds and the so-called Bayes factor. The Bayes factor turns out to be 1.05 for the January - March/snowdrop case, 2.19 for February - April/cherry, and 3.26 for the March - May/lime tree combination. In the absence of qualified prior information we choose $p\left(M_{i} \mid I\right)=1 / 2$ and the Bayes factor equals the posterior odds. The dependence of blossom onset on the evolution of the characteristic temperature is therefore, obviously reflected in the late springtime indicator (lime tree), clearly present in the case of cherry blossom, and absent in the early springtime indicator (snowdrop). The conversion from the odds ratios $O_{1 k}$ to the respective model probabilities $w_{k}$ is given by $w_{k}=O_{1 k} / \sum_{k} O_{1 k}$ with $O_{11}=1$.

\section{POOLING INFORMATION}

The results of the last section mean that coherent treatment of the temperature and blossom data is equally good (snowdrops) or better than assuming independent matching points for the models of the two data sets. We, therefore, expect that the matching point probability derived from two data sets will be more informative (e.g. better localized in time than that obtained from a single series of data). Moreover, it suggests, that also posterior estimates of trends and functional behaviour should be better in the sense of being more precise than those based on only one of the data sets. In obvious generalization of (11) we need to calculate $p\left(E \mid \vec{d}_{1}, \vec{d}_{2}, \vec{x}_{1}, \vec{x}_{2}, I\right)$ for which we need the marginal likelihood $p\left(\vec{d}_{1}, \vec{d}_{2} \mid \vec{x}_{1}, \vec{x}_{2}, E, I\right)$. This is obtained by generalization of $(6)$.

$$
\begin{aligned}
& p\left(\vec{d}_{1}, \vec{d}_{2} \mid \vec{x}_{1}, \vec{x}_{2}, E, I\right)= \\
& \int d \sigma_{1} d \sigma_{2} d \vec{f}_{1} d \vec{f}_{2} p\left(\sigma_{1} \mid I\right) p\left(\sigma_{2} \mid I\right) \cdot p\left(\overrightarrow{f_{1}} \mid I\right) \cdot p\left(\vec{f}_{2} \mid I\right) \\
& \cdot p\left(\vec{d}_{1}, \vec{d}_{2} \mid \vec{x}_{1}, \vec{x}_{2}, \vec{f}_{1}, \vec{f}_{2}, \sigma_{1}, \sigma_{2}, E, I\right) .
\end{aligned}
$$

The argument of logical independence of the data sets $\vec{d}_{1}, \vec{d}_{2}$ leads to the product expression (14) for the joint likelihood $p\left(\vec{d}_{1}, \vec{d}_{2} \mid \ldots\right)$. Since the prior probabilities factor also, the right hand side of (20) is equal to the product of the marginal likelihood of each data set separately (6). Apart from a constant factor which does not depend on $E$ explicitly (this was our previous choice of $p(E \mid I)$ ), these marginal likelihoods are equal to the matching point probabilities derived from the individual data sets. Hence,

$$
p\left(E \mid \vec{d}_{1}, \vec{d}_{2}, \vec{x}_{1}, \vec{x}_{2}, E, I\right)=p\left(\vec{d}_{1} \mid \vec{x}_{1}, E, I\right) \cdot p\left(\vec{d}_{2} \mid \vec{x}_{2}, E, I\right) / Z,
$$



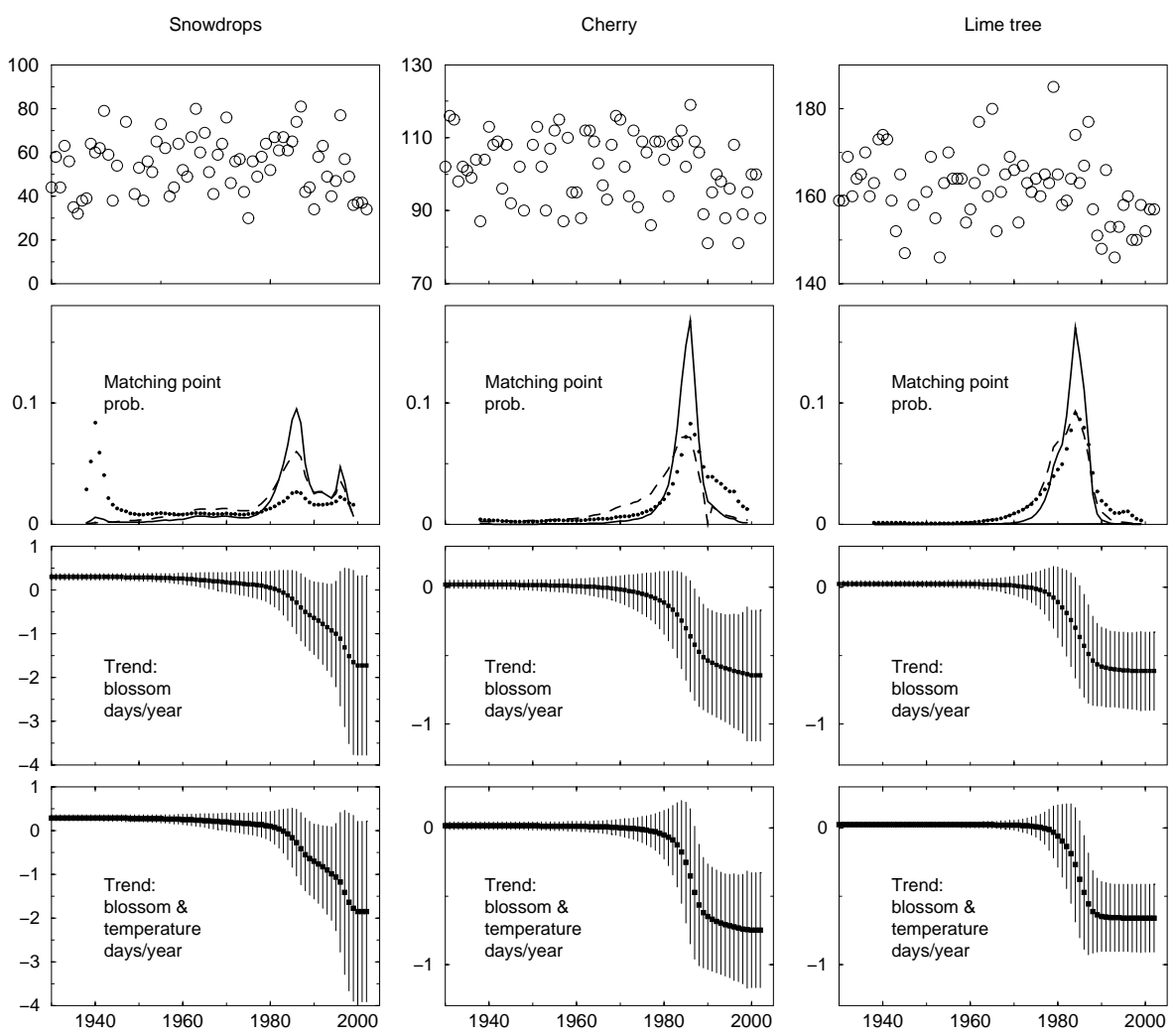

FIG. 2: The figures in the upper row show the original data on blossom onset. The second row shows matching point probabilities for the chosen two segment polygonial as obtained for the temperature data as full dots, for the blossom time series as the dashed trace and the joint probability from the pooled data set as a continuous curve. The third row reproduces blossom trend results obtained earlier on the basis of blossom time series data only (Dose 63 Menzel 2004). The lower row shows the corresponding trend predictions based on the joint temperature and blossom data sets.

where $Z$ ensures normalization. The second row of Fig. 2 shows the matching point probability of the temperature data as full dots, the matching point probability of the blossom data as the dashed trace, and the matching point probability of the joint data set as the 
continuous curve. All probability densities are normalized to unit area. As expected, we find considerably better localized distributions from the pooled data which in turn should allow more robust predictions.

Predictions based on the coherent processing of the data provide the most probable predictions, which has to be distinguished from the expected or average predictions. The latter are of course what we want. After all, an odds ration of 3:1 means that the less probable model carries still one quarter of the total probability mass, which is far from being negligible. The ambiguity is easily removed. In our previous paper (Dose \& Menzel 2004), predictions of a particular datum $D$ or functions of $D, \phi(D)$, for a particular year $z$ which need not lie within the range of the available data, were derived from the predictive distribution $p(D \mid z, \vec{d}, \vec{x}, I)$. In the present case the conditions must be changed to $\vec{d}_{1}, \vec{x}_{1}, \vec{d}_{2}, \vec{x}_{2}$ and $D$ can now be either temperature or blossom onset. The information that the two different models lead to different predictive densities must also be taken into account. We employ marginalization to account for the different models.

$$
\begin{array}{r}
p\left(D \mid z, \vec{d}_{1}, \vec{x}_{1}, \vec{d}_{2}, \vec{x}_{2}, I\right)=\sum_{M} p\left(D, M \mid z, \vec{d}_{1}, \vec{x}_{1}, \vec{d}_{2}, \vec{x}_{2}, I\right)= \\
\sum_{M} p\left(M \mid \vec{d}_{1}, \vec{x}_{1}, \vec{d}_{2}, \vec{x}_{2}, I\right) \cdot p\left(D \mid M, z, \vec{d}_{1}, \vec{x}_{1}, \vec{d}_{2}, \vec{x}_{2}, I\right)
\end{array}
$$

The first factor under the summation sign is the probability for model $M_{a, b}$ based on both data sets which is of course independent of $z$, the year of prediction. The second factor is the predictive density for $D$ given a particular model. We find that the predictive density for $D$ marginalized over the models under consideration is just their weighted average with the respective model probabilities as weights. Since the weights do not depend on $D$, equ. (21) holds also for expectation values of $\phi(D)$.

The third row of Fig. 2 displays the blossom trends obtained in this work. The bottom row shows corresponding predictions based on the joint temperature and blossom data sets. The trend changes are now in all three cases localized in the time interval from 1980 to 1990. Most interesting is the uncertainty of the predicted trend at the right extreme. For the snowdrop case, the uncertainty of the predicted trend remains unchanged if the estimate is based on both data sets instead of only one. For the lime tree case, however, we find a reduction of this uncertainty by a factor of 0.7 . This does in fact reflect directly our previous result that coherent treatment of temperature and blossom data for the lime tree case is 
superior to the incoherent treatment by a factor of 3.26 while for the snowdrop data nothing is gained by coherent treatments with the respective temperature data.

\section{DISCUSSION}

The traditional statistical analysis would quantify the relation between temperature and blossom onset by a correlation coefficient $\rho^{2}$. The correlation coefficient between a variable $y$ and a variable $x$ is a measure for the linear dependence between $x$ and $y$. The range of values of $\rho^{2}$ is $0 \leq \rho^{2} \leq 1$ with $\rho^{2}=1$ signalling perfect linear dependence. Values of $\rho^{2}<1$ arise for different reasons. If the data, neither $x$ nor $y$ are affected by noise then $\rho^{2}<1$ indicates a more complicated relationship. If the noise on the data is non-negligible, then $\rho^{2}<1$ even if the data generating mechanism is a linear relation. In general, therefore, $\rho^{2}<1$ includes both, the noise and the deviation from a linear interdependence.

The present problem may be reformulated such that the hypothesis of a linear relation makes sense. Let $T$ denote temperature, $B$ blossom onset and $t$ the year of observation. We then have a parameter representation of the relationship between blossom onset and temperature evolution

$$
B=f(t) \quad, \quad T=g(t), \Rightarrow B=f\left(g^{-1}(T)\right),
$$

where $t=g^{-1}(t)$ is the inverse to $g(t)$. The relation between $B$ and $g$ becomes a linear one if $f \sim g$ which includes the special case that both $g$ and $f$ are linear mappings.

Numerical values of the correlation coefficients $\rho^{2}$ are 0.56 for snowdrop/January-March, 0.65 for cherry/February-April and 0.63 for lime tree/March-May. For interpretation of the numbers it is important to note that we have good reason to assume the noise levels to be independent of the data combination. Variations of $\rho^{2}$ can then uniquely be attributed to different functions $f$ and $g$ in the respective cases. We find that correlation analysis also gives the weakest result for snowdrop blossom / January - March temperatures, however correlation analysis does not differentiate between cherry and lime tree in contrast to the Bayesian result. The Bayesian model comparison outlined above does not require the assumptions we need to make for a correlation analysis and comes up with a unique answer on the amount of interdependence in the respective time series pairs. For snowdrops the coherent treatment of both time series explains the data sets equally well as the incoherent 
treatment. For cherry and lime tree data the coherent option is preferred by factors of 2.2 and 3.3, respectively. Concomitant with the preference for a coherent treatment is a decrease of the uncertainty for the trend predictions in cherry and lime tree blossom based on the combined data set, in the latter case by as much as a factor of 0.7 . The origin of the gain in precision is the close similarity of the matching point distributions for the blossom and temperature data as in the upper right panel of Fig. 2. Formally, what happens may be seen by reference to equ. (2). Rewriting the term under the summation sign

$$
p(E \mid I) \cdot p\left(\vec{d}_{1} \mid \vec{x}_{1}, E, I\right) \cdot p\left(\vec{d}_{2} \mid \vec{x}_{2}, E, I\right)=p\left(E \mid \vec{d}_{1}, \vec{x}_{1}, I\right) \cdot p\left(\vec{d}_{2} \mid \vec{x}_{2}, E, I\right)
$$

shows that the coherent treatment is equivalent to employing the posterior probability of $E$ given data set $\vec{d}_{1}$ (up to a constant $p\left(\vec{d}_{1} \mid \vec{x}_{1}, I\right)$ ) from the evaluation of data set $\vec{d}_{1}$ as a prior probability for the analysis of the data set $\vec{d}_{2}$. Since this prior is informative, it has an influence on the probability distribution of $E$ based on the prior and data set $\vec{d}_{2}$. In fact, $p\left(E \mid \vec{d}_{1}, \vec{x}_{1}, \vec{d}_{2}, \vec{x}_{1}, I\right)$ is narrower than $p\left(E \mid \vec{d}_{2}, \vec{x}_{2}, I\right)$ and, therefore, the changes in the trends become more localized which in turn is reflected in more precise predictions.

From the biological point of view, the assessment of impacts of climate change on systems, such as phenology (in our case flowering dates), requires a most rigorous change detection and the attribution to climate change. Bayesian analysis for change detection in phenological time series had been introduced by Dose \& Menzel (2004); that earlier study revealed for three flowering records (also subject of this paper) that the model used in this paper was the most likely one. It also exhibited maximum matching point probabilities in the mid 1980s (see also second row of Fig. 2. Snowdrop blossom advanced by -1.5 days / year in 2002, cherry and lime tree blossom by -0.6 days / year (2002).

From literature it is well known that springtime phenological phases are particularly sensitive to temperature, thus phenology seems to be an ideal bio-indicator (see e.g. review by Walther et al 2002). Spring temperatures at Geisenheim, which correlate with the respective blossom time series, increased by $0.1^{\circ} \mathrm{C} /$ year (January - March), $0.15^{\circ} \mathrm{C} /$ year (February - April) and $0.09^{\circ} \mathrm{C} /$ year (March - May).

Within the IPCC report (IPCC 2001) or in recent meta-analyzes (e.g. by Root et al. 2003), Parmesan \& Yohe 2003), changes in both phenological and temperature time series and their correlation would have resulted in a judgement of observed climate change impact.

The presented Bayesian analysis provides further insight in the amount of interdepen- 
dence in the respective time series. First of all, it is of tremendous importance that the observed "discontinuity" of the phenological records in Central Europe in the mid 1980s is, at least for cherry and lime tree blossom, strongly supported by the respective mean spring temperatures. The reasons for the specific location of the matching points in the mid 1980s are speculative; most likely, they are connected to altered atmospheric circulation patterns, such as the North Atlantic Oscillation (NAO) (e.g. Menzel 2003).

Quantitative differences in the Bayes factors of cherry and lime tree, reflecting different amounts of interdependence, are not evident in traditional correlation analysis. These differences between cherry and lime tree results may be related to different temperature sensitivities, which will be analyzed in a next step.

The fact that no preference for a coherent treatment of snowdrop blossom and January March temperatures was found, although traditional correlation analysis gives a correlation coefficient $\rho^{2}$ of 0.56 , should raise further questions. Either January - March temperatures are not the most appropriate measure to attribute observed changes in snowdrop blossom to, and other possibly triggering parameters, such as soil temperatures or snow cover should be considered, too. Or changes in blossom dates must be (partly) attributed to other causes, such as changes in variety (e.g. Menzel et al. 2006) or microclimatic conditions at top soil / ground level which are not mirrored by air temperature measured at $2 \mathrm{~m}$ height.

Thus, the Bayesian answer to the question whether changes in temperature and phenology should be regarded as coherent or independent also offers deeper biological insight.

In conclusion, we find that the Bayesian treatment of the problem does not only provide us with numbers of well defined meaning as to which of the two alternative hypotheses $M_{a}$ : the evolution of temperature and blossom onset time series is causally independent and $M_{b}$ : the two time series exhibit correlation, is supported by the data. In case that $M_{b}$ dominates it allows also pooling of temperature and blossom data to arrive at much more precise trend estimates. The return of the Bayesian analysis is therefore much superior to the results of a traditional correlation analysis. 
References

Boyer WD (1973) Air temperature, heat sums, and pollen shedding phenology of longleaf pine. Ecology, 54, 421-425.

Cannell MGR \& Smith RI (1983) Thermal time, chill days and prediction of budburst in Picea sitchensis. Journal of Applied Ecology, 20 (3), 951-963.

Chuine I (2000) A unified model for the budburst of trees. Journal of Theoretical Biology, 204 (3), 337-347.

Chuine I, Kramer K \& Hänninen H (2003) Plant development models. In: Schwartz MD:

Phenology: An integrative environmental science. Kluwer Academic Publishers, Dordrecht, Boston, London.

Dose V, Menzel A (2004) Bayesian analysis of climate change impacts in phenology. Global Change Biology, 10, 259-272.

Emberlin J, Mullins J, Cordon J, Millington W, Brooke M, Savage M \& Jones S (1997) The trend to earlier Birch pollen season in the U.K.: a biotic response to changes in weather conditions? Grana, 36, 29-33.

Fitter AH \& Fitter RSR (2002) Rapid change in flowering time in British plants. Science, 296, 1689-1691.

Gregory PC (2005) Bayesian Logical Data Analysis for the Physical Sciences; A Comparative Approach with Mathematical Support. Cambridge University Press, Boston.

Hänninen H (1987) Effects of temperature on dormancy release in woody plants: implications of prevailing models. Silva Fennica, 21, 279-299.

Hänninen H (1990) Modelling bud dormancy release in trees from cool and temperate regions. Acta Forestalia Fennica, 213, 1-47.

Hänninen H, Kellomäki S, Laitinen K, Pajari B, Repo T (1993) Effect of increased winter temperature on the onset of height growth of Scots pine: a field test of a phenological model. Silva Fennica, 27, 251-257.

IPCC (2001) Climate change 2001: The scientific basis. In: Third Assessment report of the Working Group I (eds Houghton JT et. al), Cambridge University Press, Cambridge. IPCC (2001) Climate change 2001: Impacts, Adaptation and Vulnerability. In: Impacts, adaptation $\&$ vulnerability, contributions of Working Group II to the Third Assessment Report of the Intergovernmental Panel on Climate Change (eds McCarthy JJ et al.), Cambridge University Press, Cambridge. 
Jaynes ET (2003) Probability Theory The Logic of Science (edited by G. Larry Bretthorst). Cambridge University Press.

Jaynes ET (1957) Information theory and statistical mechanics. Physical Review, 106, 620-630. Jeffreys H (1961) Theory of Probability. Oxford University Press.

Kapur JN, Kesavan HK (1992) Entropy Optimization Principles with Applications. Academic, Boston.

Kramer K (1994) Selecting a model to predict the onset of growth of Fagus sylvatica. Journal of Applied Ecology, 31 (1), 172-181.

Landsberg JJ (1974) Apple fruit bud development and growth: analysis and an empirical model. Annals of Botany, 38 (158), 1013-1023.

Linkosalo T, Carter TR, Häkkinen R \& Hari P (2000) Predicting spring phenology and frost damage risk of Betula sp. under climatic warming: a comparison of two models. Tree Physiology, 20, 1175-1182.

Menzel A (2002) Phenology: its importance to the global change community. Climatic Change, 54, 379-385.

Menzel A (2003) Plant phenological anomalies in Germany and their relation to air temperature and NAO. Climatic Change, 57 (3), 243-263.

Menzel A, Estrella N, Testka A (2006) Temperature response rates in long-term phenological records. Climatic Research, in press.

Murray MB, Cannell MGR, Smith RI (1989) Date of budburst of fifteen tree species in Britain following climatic warming. Journal of Applied Ecology, 26, 693-700.

Parmesan C, Yohe G (2003) A globally coherent fingerprint of climate change impacts across natural systems. Nature, 421, 37-42.

Reaumur RAF de (1735) Observations du thermomètre, faites a Paris pendant l'année 1735, comparées avec celles qui ont été faites sous la ligne, à l'isle de France, à Alger et quelques unes de nos isles de l'Amérique. Memoires de l'Académie des Sciences de Paris.

Root TL, Price JT, Hall KR, Schneider SH, Rosenzweig C \& Pounds JA (2003) Fingerprints of global warming on wild animals and plants. Nature, 421, 57-60.

Schaber J, Badeck FW (2003) Physiology-based phenology models for forest tree species in Germany. International Journal of Biometeorology, 47, 193-201.

Schnelle F (1955) Pflanzenphänologie. Akademische Verlagsgesellschaft Geest und Portig, Leipzig. 
Schwartz MD (1997) Spring Index Models: An approach to connecting satellite and surface phenology. In: Phenology of Seasonal Climates I, edited by H. Lieth and MD Schwartz, 23-38. Backhuys, Netherlands.

Sivia DS (1996) Data Analysis: A Bayesian Tutorial. Clarendon Press, Oxford.

Sparks TH, Jeffree EP \& Jeffree CE (2000) An examination of the relationship between flowering times and temperature at the national scale using long-term phenological records from the U.K. International Journal of Biometeorology, 44, 82-87.

Sparks TH, Carey PD (1995) The responses of species to climate over 2 centuries - an analysis of the Marsham phenological record, 1736-1947. Journal of Ecology, 83 (2), 321-329.

Walther GR, Post E, Convey P et al. (2002) Ecological responses to recent climate change. Nature, 416, 389-395. 